\title{
Bicicleta o Angioplastia en angina crónica estable
}

\section{Objetivo}

Comparar la efectividad del entrenamiento físico supervisado vs. angioplastia con stent en pacientes con angina crónica estable (ACE).

\section{Pacientes}

Se incorporaron 101 hombres con ACE, menores de 70 años y por lo menos una arteria coronaria con estenosis $>75 \%$ plausible de ser revascularizada con angioplastia. Todos los pacientes tenian angina en clase funcional I- II-III e isquemia documentada en pruebas evocadoras de isquemia (PEI). Los pacientes fueron seguidos por 12 meses.

\section{Diseño}

Ensayo clínico aleatorizado siendo los médicos evaluadores de los resultados ciegos a la intervención inicial.

\section{Lugar}

Centro de Cardiología de la Universidad de Leipzig, Alemania

\section{Medición de resultados principales}

Se realizaron mediciones iniciales y al año de la clase funcional de la angina así como de PEI para medir cambios en el umbral isquémico, consumo de oxígeno, y capacidad de ejercicio. Además se realizaron estudios de perfusión y CCG basales y a los 12 meses

\section{Intervención}

Los pacientes fueron aleatorizados a angioplastia con stent ó programa supervisado de actividad física. La angioplastia se realizó 14.8 días posteriores a la randomización y el comienzo del ejercicio supervisado 21.3 días. En el programa de ejercicio supervisado se les proveía a los pacientes de una bicicleta ergométrica y se los instruía para alcanzar la meta del $70 \%$ de la frecuencia cardíaca máxima obtenida durante la PEI positiva. La indicación de ejercicio eran diariamente durante 20 minutos y una vez a la semana una sesión grupal de 60 minutos de ejercicio aeróbico.

\section{Resultados}

El análisis fue por intención de tratar. Los grupos fueron similares en cuanto a características basales y de tratamiento.Ocurrieron 21 eventos en total, 15 en el grupo angioplastia y seis en el grupo ejercicio. Las curvas de sobrevida libre de eventos al año fueron significativamente diferentes, $70 \%$ vs. $88 \%$ respectivamente. En ambos grupos los síntomas mejoraron significativamente. En el grupo ejercicio la clase funcional inicial mejoró de un 1.5 inicial a 0.4 al fina de los 12 meses mientras que en el grupo angioplastia varió de 1.7 inicial a 0.7 a los 12 meses.

Resultados al final del estudio variables ergométricas

\begin{tabular}{l|c|c|c} 
& Grupo ejercicio & $\begin{array}{c}\text { Grupo } \\
\text { angioplastia }\end{array}$ & Valor de p \\
\hline Frecuencia cardiaca de reposo & 65 & 70 & $<0.005$ \\
\hline Umbral isquémico (W) & 127 & 119 & $<0.005$ \\
\hline Capacidad f́sica (W) & 159 & 130 & $<0.005$ \\
\hline
\end{tabular}

El promedio de un año de costos por paciente del grupo ejercicio fue de U\$S 3708 comparado con U\$S 6086 del grupo angioplastia. Para cambiar una clase funcional de angina se gastaron U\$S 3429 en el grupo ejercicio y U\$S 6959 en el grupo angioplastia.

\section{Conclusión}

En pacientes con angina crónica estable un programa de ejercicios supervisados de 12 meses demostró ser más efectivo y de menor costo que la angioplastia con STENT

\section{Comentario}

Existen tres estudios que compararon angioplastia con tratamiento médico en ACE en los que se observa que el tratamiento invasivo no logra disminuir ni la mortalidad cardiovascular ni el infarto no fatal, inclusive en dos de ellos la sobrevida acumulada libre de eventos fué menor en el grupo asignado a tratamiento médico ${ }^{1,2,3}$. Esto puede explicarse por los riesgos relacionados con el procedimiento y que los pacientes incluidos eran de bajo riesgo. La angioplastia demostró mejorar la calidad de vida al disminuir significativamente la clase funcional de la angina y la utilización de drogas antianginosas. La limitación de estos estudios es no haber utilizado stents, que se relacionan con menor reestenosis y que pocos pacientes se encontraban bajo tratamiento hipolipemiante. Un cuarto estudio, el AVERT, ya en la "era de las estatinas" comparó angioplastia con stent vs. tratamiento con atorvastatina a altas dosis, el resultado fue favorable al tratamiento médico en un resultado combinado cuyas diferencias en términos absolutos eran muy pequeñas ${ }^{4}$. Un metaanálisis reciente en pacientes coronarios mostró en aquellos que realizaban actividad física una disminución del $31 \%$ en la mortalidad cardioavscular ${ }^{5}$

Los resultados del estudio comentado en esta edición de Evidencia muestran una diferencia a favor de un programa de actividad física con respecto a angioplastia con stent. Lo que añade este estudio es que la intervención sobre la actividad fisica mejora de igual manera que la angioplastia la clase funcional de la angina y la supera en cuanto a disminuir el umbral de angina. El mecanismo del beneficio es muy distinto ya que no se produce por una reducción de la estenosis coronaria si no por disminución de la disfunción endotelial lo que implica una mejoría en todo el árbol vascular.La intervención indicada si bien debería ser probada en estudios más grandes y multicéntricos es fácil de hacer y de bajo costo (inclusive podría ser menor en latino américa dado el bajo costo laboral y el hecho de importar stents), pero habría que evaluar su reproducibilidad. El problema sería ciertos aspectos anacrónicos en el contexto de una sociedad empobrecida, donde es más fácil colocar un stent que trabajar sobre la motivación, hacer que los pacientes dejen de fumar, hagan actividad física y disminuyan el colesterol con estatinas. Siempre debemos pensar en el costo de oportunidad, que dejamos hacer cuando elegimos una intervención. Por definición del último consenso del ACC/AHA considera éxito terapéutico en la ACE a la "mejoría completa de la angina sin padecer efectos adversos de las drogas que limiten la calidad de vida, teniendo en cuenta las expectativas y preferencias individuales de cada paciente ${ }^{-6}$. Esto implica el desarrollo de capacidades para realizar evaluaciones clínicas y funcionales, que permitan decidir la revascularización en el momento adecuado según la sitruación particular de cada paciente.

Conclusiones del comentador: Un programa de actividad física parece ser superior a la angioplastia con stent, este resultado presisa ser confirmado en estudios multicéntricos así como la validez externa de la intervención ya que requiere de médicos y pacientes motivados.

Dr. Augusto Granel [ Unidad de Medicina Familiar y Preventiva. Hospital Italiano de Buenos Aires.]

Granel A. Bicicleta o Angioplastia en angina crónica estable. Evid. actual. práct. ambul. 2004;7:132. Comentado de: Hambreth R, Walther C, Sven M, et al. Percutaneous Coronary Angioplasty Compared with Exercise Training in patients with Stable Coronary Artery Disease. Circulation 2004;109: 1371-1378

Referencias

1.Parisi A, Folland DE Hartigan P. A comparison of angioplasty with medical therapy in the treatment of single vessel coronary artery disease (ACME). N Engl J Med 1992; 326: 10-16 2.Huet W, Belotti A, Oliveira SA. The medicine, angioplasty or surgery study (MASS): A prospective randomized trial of medical therapy, angioplasy or by pass surgery for single proximal descending artery stenosis. Am Coll Cardiol 1995; 26:1600-5

3.Rita 2 trial participants. Coronary angioplasty versus medical therapy for angina: the sedond randomized intervention treatment of angina. Lancet 1997; 350:461-8 4. Pitt B, Raers D, Brown W. Aggresive lipid lowering treatment compared with angioplasty in stable coronary disease.N Engl J Med 1992; 326: 10-16

5.Joliffe JA, Rees K, Taylor et al. exrcide based rehabilitation for coronary disease. The Cochrane Library, isue 4, 2002, Oxford, UK.

6.Gibbons et al.AHA/ACC. Guideline update for the management of patients with chronic stable angina. Executive summary and recommendations. Circulation 2003 